\title{
automatica
}

PERGAMON

\section{An exponential stability result for the wave equation ts}

\author{
Ömer Morgül * \\ Bilkent University, Dept. of Electrical and Electronics Engineering, 06533, Bilkent, Ankara, Turkey
}

Received 27 March 2000; received in revised form 8 December 2000; accepted 9 January 2001

\begin{abstract}
We consider a system described by the one-dimensional linear wave equation in a bounded domain with appropriate boundary conditions. To stabilize this system, we propose a dynamic boundary controller applied at the free end of the system. The transfer function of the proposed controller is a proper rational function which consists of a strictly positive real function and some poles on the imaginary axis. We then show that under some conditions the closed-loop system is exponentially stable. (C) 2002 Published by Elsevier Science Ltd.
\end{abstract}

Keywords: Distributed parameter systems; Control theory; Stability

\section{Introduction}

In recent years, boundary control of infinite-dimensional systems has become an important research area, see e.g. Luo, Guo, and Morgül (1999) for more information and references. In this note, we will consider a system described by the one-dimensional wave equation in a bounded domain. We assume that a dynamic boundary control is applied to the system for stabilization. We propose a (rational) controller transfer function, which contains a strictly positive real part and some simple poles on the imaginary axis. The residues associated with the imaginary axis poles are assumed to be positive. Such transfer functions have been proposed to stabilize the wave equation, see Morgül (1994, 1998), where it was shown that with these controllers, the resulting closed-loop system is asymptotically stable under some conditions. In many cases, exponential stability is desired, due to e.g. the robustness of the resulting closed-loop system, and in infinite-dimensional systems, asymptotic stability may not imply exponential stability.

Note that exponential stability for this system could be achieved by static output feedback, see Chen (1979). However, if we also want to achieve tracking and/or disturbance rejection for certain classes of output signals,

\footnotetext{
This paper was not presented at any IFAC meeting. This paper was recommended for publication in revised form by Associate Editor Rodolphe Sepulchre under the direction of Editor Paul Van den Hof.

* Corresponding author. Fax: +90-312-2664192.

E-mail address: morgul@ee.bilkent.edu.tr (Ö. Morgül).
}

then we need a dynamic controller containing an internal model, see Hämäläinen and Pohjolainen (2000), Morgül (1998). The dynamic controllers discussed in this paper would be suitable for reference and disturbance signals which are the superposition of a constant and a sinusoid at frequency $\omega_{1}$. We show that the resulting closed-loop system is exponentially stable under some conditions. We do not discuss the tracking error.

\section{Problem statement}

In this note, we consider the following system:

$u_{t t}(x, t)=u_{x x}(x, t)$

$u(0, t)=0, \quad u_{x}(1, t)=-f(t)$,

where, without the loss of generality various coefficients, including the length of the spatial domain, are assumed to have unit values, $x \in(0,1)$ denotes the spatial variable, $t \geqslant 0$ denotes time, $u(\sigma, \tau)$ denotes the solution of the wave equation at $x=\sigma, t=\tau$, a subscript as in $u_{t}$ denotes the partial derivative with respect to the corresponding variable, and $f: \mathbf{R}_{+} \rightarrow \mathbf{R}$ is the boundary control applied at the end point $x=1$. The systems whose behaviour may be modelled by (1)-(2) include strings, vibrations of long cables, longitudinal motion and torsional vibrations of flexible beams, etc. 
It is well-known that if we use the following controller:

$f(t)=d u_{t}(1, t), \quad d \geqslant 0$

then the resulting closed-loop system is exponentially stable in an appropriate Hilbert space, see Chen (1979). The static controllers given by (3) were extended to $d y$ namic ones in Morgül (1994, 1998), i.e. the controller is a finite-dimensional linear time-invariant (LTI) system whose input and output are given by $u_{t}(1, t)$ and $f(t)$, respectively. Following Morgül (1994, 1998), we propose the following model for the controller:

$\dot{z}_{1}=A z_{1}+b u_{t}(1, t), \quad \dot{x}_{1}=\omega_{1} x_{2}$,

$\dot{x}_{2}=-\omega_{1} x_{1}+u_{t}(1, t)$,

$f(t)=c^{\mathrm{T}} z_{1}+d u_{t}(1, t)+k u(1, t)+k_{1} x_{2}$,

where $z_{1} \in \mathbf{R}^{n}$, for some natural number $n, A \in \mathbf{R}^{n \times n}$ is a constant matrix, $b, c \in \mathbf{R}^{n}$ are constant column vectors, $\omega_{1}, d, k, k_{1} \in \mathbf{R}$, are various non-negative constants and the superscript $\mathrm{T}$ denotes transpose. If we take the Laplace transform in (4)-(5), we obtain the following transfer function $h(s)$ for the controller:

$h(s)=h_{1}(s)+\frac{k}{s}+\frac{k_{1} s}{s^{2}+\omega_{1}^{2}}$,

where $h_{1}(s)=c^{\mathrm{T}}(s I-A)^{-1} b+d$, i.e. we have $\hat{f}(s)=$ $h(s) \hat{u}_{t}(1, s)$, where a hat denotes the Laplace transform of the corresponding variable. It was shown in Morgül (1998) that this type of controllers are useful for disturbance rejection. Following Morgül (1994, 1998), we assume that: (i) $A$ is stable, (ii) the triplet $(A, b, c)$ is minimal, that is $(A, b)$ is controllable and $(A, c)$ is observable, see e.g. Curtain and Zwart (1995) and (iii) for some $\gamma \geqslant 0$ we have

$\mathfrak{R}\left\{h_{1}(\mathrm{j} \omega)\right\}>\gamma, \omega \in \mathbf{R}$.

We first define the following function spaces:

$$
\mathbf{H}=\left\{\left(\begin{array}{lllll}
u & v & z_{1} & x_{1} & x_{2}
\end{array}\right)^{\mathrm{T}} \mid u \in \mathbf{H}_{0}^{1}, v \in \mathbf{L}^{2}, z_{1} \in \mathbf{R}^{n}, x_{1}, x_{2} \in \mathbf{R}\right\},
$$

$\mathbf{L}^{2}=\left\{f:[0,1] \rightarrow \mathbf{R} \mid \int_{0}^{1} f^{2} \mathrm{~d} x<\infty\right\}$,

$\mathbf{H}_{0}^{k}=\left\{f \in \mathbf{L}^{2} \mid f, f^{\prime}, \ldots, f^{(k)} \in \mathbf{L}^{2}, f(0)=0\right\}$.

Note that in (9) $f$ is measurable, and in (10), $f, f^{\prime}, \cdot, f^{(k-1)}$ are absolutely continuous. Eqs. (1)-(2), (4)-(5) can be given as follows:

$\dot{z}=L z, \quad z(0) \in \mathbf{H}$, where $z=\left(\begin{array}{lllll}u & u_{t} & z_{1} & x_{1} & x_{2}\end{array}\right)^{\mathrm{T}} \in \mathbf{H}$, the operator $L: \mathbf{H} \rightarrow \mathbf{H}$ and its domain $D(L)$ are defined as

$$
\begin{aligned}
& L\left(\begin{array}{c}
u \\
v \\
z_{1} \\
x_{1} \\
x_{2}
\end{array}\right)=\left(\begin{array}{c}
v \\
u_{x x} \\
A z_{1}+b v(1) \\
\omega_{1} x_{2} \\
-\omega_{1} x_{1}+v(1)
\end{array}\right) . \\
& D(L):=\left\{\left(\begin{array}{lllll}
u & v & z_{1} & x_{1} & x_{2}
\end{array}\right)^{\mathrm{T}} \in \mathbf{H} \mid u \in \mathbf{H}_{0}^{2}, v \in \mathbf{H}_{0}^{1}, z_{1} \in \mathbf{R}^{n},\right. \\
& x_{1}, x_{2} \in \mathbf{R} ; u_{x}(1)+c^{\mathrm{T}} z_{1}+\mathrm{d} v(1) \\
& \left.+k u(1)+k_{1} x_{2}=0\right\} \text {. }
\end{aligned}
$$

In $\mathbf{H}$, we define the following "energy" norm:

$$
\begin{aligned}
\|z\|_{E}^{2}= & \frac{1}{2}\left(\int_{0}^{1}\left(v^{2}+u_{x}^{2}\right) \mathrm{d} x+k u^{2}(1, t)+z_{1}^{\mathrm{T}} P z_{1}\right. \\
& \left.+k_{1}\left(x_{1}^{2}+x_{2}^{2}\right)\right),
\end{aligned}
$$

where $P$ is an appropriate symmetric, positive definite matrix, see Morgül $(1994,1998)$. We note that the norm given above is induced by an appropriate inner-product, hence $\mathbf{H}$ is a Hilbert space. Note that in the following, we may work in the complexified versions of these Hilbert spaces, but for convenience we do not change the notation. Next we summarize the results of Morgül (1994, 1998).

Theorem 1. Consider the system given by (11) and let the assumptions stated above hold.

(i) The operator $L$ generates a $C_{0}$ semigroup of contractions on $\mathbf{H}$ (for the terminology of semigroup theory, see e.g. Luo, Guo, \& Morgül, 1999).

(ii) If $k_{1} \geqslant 0$ and $\omega_{1} \neq m \pi$ for all natural numbers $m$, then $T(t)$ is asymptotically stable.

(iii) If $k_{1}=0$ and $\gamma>0$ (see (7)), then $T(t)$ is exponentially stable.

Proof. For (i) and (ii), see Morgül (1998), and for (iii), see Morgül (1994).

It was conjectured in Morgül (1998) that even if $k_{1}>0$, we may have exponential stability, provided that $\gamma>0$. In the sequel, we will prove this statement. We will need the following result, which is due to F.L. Huang, see e.g. Luo, Guo, and Morgül (1999).

Theorem 2. Let $T(t)$ be a bounded $C_{0}$-semigroup generated by an operator $A$, in a Hilbert space. Then, $T(t)$ is exponentially stable if and only if the imaginary axis belongs to the resolvent set of $A$, and the following holds:

$\sup _{\omega \in \mathbf{R}}\left\|(\mathrm{j} \omega I-A)^{-1}\right\|<\infty$. 


\section{Exponential stability}

Our main result is the following:

Theorem 3. Consider the system given by (11). Let the assumptions stated above hold, and let $\gamma>0$, (see (7)). If $\omega_{1} \neq m \pi$ for all natural numbers $m$, then $T(t)$ is exponentially stable.

Proof. The case $k_{1}=0$ was proven in Morgül (1994), hence we consider the case $k_{1}>0$. Note that the operator $(\lambda I-L)^{-1}: \mathbf{H} \rightarrow \mathbf{H}$ is compact for $\lambda \geqslant 0$; hence, the spectrum of $L$ consists entirely of isolated eigenvalues and moreover $\lambda=0$ is not an eigenvalue of $L$, see Morgül (1998).

In the proof, we will use Theorem 2 stated above. First note that by Theorem $1,\|T(t)\|$ is bounded. Next, we will show that the imaginary axis belongs to the resolvent set of $L$. By contradiction, assume that for some $\omega \in \mathbf{R}, \mathrm{j} \omega$ does not belong to the resolvent set of $L$. Since $L$ has compact resolvent, it follows that for some non-zero $z \in D(L)$, we must have

$(\mathrm{j} \omega I-L) z=0$.

First assume that $\omega \neq \omega_{1}$. By using (12) in (16) and $z=$ $\left(\begin{array}{lllll}u & v & z_{1} & x_{1} & x_{2}\end{array}\right)^{\mathrm{T}} \in D(L)$, we obtain

$u(x)=C \sin \omega x, \quad v(x)=\mathrm{j} \omega C \sin \omega x$,

$z_{1}=(\mathrm{j} \omega I-A)^{-1} b v(1), \quad\left((\mathrm{j} \omega)^{2}+\omega_{1}^{2}\right) x_{2}=\mathrm{j} \omega v(1)$,

where $C \neq 0$ is a constant. By using (17)-(18) in (13) we obtain

$C \omega(\cos \omega+\mathrm{j} h(\mathrm{j} \omega) \sin \omega)=0$,

where $h(s)$ is given by (6). Let us define $h(\mathrm{j} \omega)=R(\omega)+$ $\mathrm{j} I(\omega)$ where $R(\omega)$ and $I(\omega)$ are real and imaginary parts of $h(\mathrm{j} \omega)$, respectively. Note that $s=0$ is not an eigenvalue, hence $\omega \neq 0$. Hence from (19) we obtain

$R(\omega) \sin \omega=0, \quad \cos \omega-I(\omega) \sin \omega=0$.

Since $R(\omega)>\gamma>0$, from (20) we obtain $\sin \omega=0$ and $\cos \omega=0$, which is a contradiction. If $\omega=\omega_{1}$, from (18) we obtain $v(1)=0$ and from (17) we obtain $\sin \omega_{1}=0$, which implies $\omega_{1}=m \pi$ for some $m$, contradicting our assumption. Hence, it follows that the imaginary axis belongs to the resolvent set of $L$.

Finally, to show that (15) holds, note that since $(\mathrm{j} \omega I-L)^{-1}$ is a holomorphic function on the resolvent set, see e.g. Curtain and Zwart (1995), it follows that for any $\Omega>0$, the following holds:

$\sup \left\|(\mathrm{j} \omega I-L)^{-1}\right\|_{E}<\infty$, where the norm in (21) is the operator norm induced by (14). Hence, to prove (15), it suffices to consider the behaviour of the resolvent as $\omega \rightarrow \infty$.

Let $\omega$ be sufficiently large. Let $y=\left(\begin{array}{llll}p q & r & r_{1} & r_{2}\end{array}\right)^{\mathrm{T}} \in \mathbf{H}$ be given and let $z=\left(\begin{array}{lllll}u & v & z_{1} & x_{1} & x_{2}\end{array}\right)^{\mathrm{T}} \in D(L)$ be such that the following holds:

$(\mathrm{j} \omega I-L) z=y$.

By using (12), (22) can be rewritten as follows:

$$
\begin{aligned}
& \mathrm{j} \omega u-v=p, \quad \mathrm{j} \omega v-u_{x x}=q, \\
& (\mathrm{j} \omega I-A) z_{1}-b v(1)=r, \\
& \mathrm{j} \omega x_{1}-\omega_{1} x_{2}=r_{1}, \quad \mathrm{j} \omega x_{2}+\omega_{1} x_{1}-v(1)=r_{2} .
\end{aligned}
$$

From the last equation in (23) we obtain

$z_{1}=(\mathrm{j} \omega I-A)^{-1}(b v(1)+r)$.

By using the first two equations in (23), we obtain a differential equation in $u$, whose solution satisfying $u(0)=0$ is given by

$u(x)=\mathrm{j} C \sin \omega x-\frac{1}{\omega} \int_{0}^{x}[q(\sigma)+\mathrm{j} \omega p(\sigma)] \sin \omega(x-\sigma) \mathrm{d} \sigma$,

where $C$ is a constant to be determined by the second line in (13). By using (23) and (26) in (13) we obtain

$\mathrm{j} \omega C(\cos \omega+\mathrm{j} h(\mathrm{j} \omega) \sin \omega)=K$,

$$
\begin{aligned}
K= & \int_{0}^{1}(q(\sigma)+\mathrm{j} \omega p(\sigma))(\cos \omega(1-\sigma)+\mathrm{j} h(\mathrm{j} \omega) \\
& \sin \omega(1-\sigma)) \mathrm{d} \sigma+\left[h(\mathrm{j} \omega)-\frac{k}{\mathrm{j} \omega}\right] p(1) \\
& -c^{\mathrm{T}}(\mathrm{j} \omega I-A)^{-1} r+k_{1} \frac{\omega_{1} r_{1}-\mathrm{j} \omega r_{2}}{(\mathrm{j} \omega)^{2}+\omega_{1}^{2}},
\end{aligned}
$$

where $h(s)$ is given by (6). Using integration by parts we obtain

$\omega \int_{0}^{x} p(\sigma) \cos \omega(x-\sigma) \mathrm{d} \sigma=\int_{0}^{x} p^{\prime}(\sigma) \sin \omega(x-\sigma) \mathrm{d} \sigma$

$$
\begin{aligned}
& \omega \int_{0}^{x} p(\sigma) \sin \omega(x-\sigma) \mathrm{d} \sigma \\
& \quad=p(x)-\int_{0}^{x} p^{\prime}(\sigma) \cos \omega(x-\sigma) \mathrm{d} \sigma .
\end{aligned}
$$

Moreover, for any $p \in \mathbf{H}_{0}^{1}$ we have (see Morgül, 1994)

$|p(x)|^{2} \leqslant \int_{0}^{x}\left|p^{\prime}(\sigma)\right|^{2} \mathrm{~d} \sigma$. 
By using (23), (26), (28)-(31), we obtain

$$
\begin{aligned}
& \int_{0}^{1}\left|u^{\prime}(\sigma)\right|^{2} \mathrm{~d} \sigma \\
& \leqslant 3\left(|\omega C|^{2}+\int_{0}^{1}|q(\sigma)|^{2} \mathrm{~d} \sigma+\int_{0}^{1}\left|p^{\prime}(\sigma)\right|^{2} \mathrm{~d} \sigma\right), \\
& \int_{0}^{1}|v(\sigma)|^{2} \mathrm{~d} \sigma \\
& \leqslant 3\left(|\omega C|^{2}+\int_{0}^{1}|q(\sigma)|^{2} \mathrm{~d} \sigma+\int_{0}^{1}\left|p^{\prime}(\sigma)\right|^{2} \mathrm{~d} \sigma\right),
\end{aligned}
$$

By using (23), (26), (30), and (31) in (25) we obtain

$$
\begin{gathered}
\left\|z_{1}\right\|^{2} \leqslant K_{1}\left\|(\mathrm{j} \omega I-A)^{-1}\right\|^{2} \\
\left(|\omega C|^{2}+\int_{0}^{1}|q(\sigma)|^{2} \mathrm{~d} \sigma\right. \\
\left.+\int_{0}^{1}\left|p^{\prime}(\sigma)\right|^{2} \mathrm{~d} \sigma+\|r\|^{2}\right),
\end{gathered}
$$

where $K_{1}$ is an appropriate constant. Finally, note that

$$
\begin{aligned}
|D(\omega)|^{2} & =|\cos \omega+\mathrm{j} h(\mathrm{j} \omega) \sin \omega|^{2} \\
& =|\cos \omega-I(\omega) \sin \omega+\mathrm{j} R(\omega) \sin \omega|^{2} \\
& \geqslant \gamma^{2} \sin ^{2} \omega+\cos ^{2} \omega-I(\omega) \sin 2 \omega .
\end{aligned}
$$

Note that $I(\omega) \rightarrow 0$ as $\omega \rightarrow \infty$, and $\gamma^{2} \sin ^{2} \omega+$ $\cos ^{2} \omega \geqslant \min \left\{\gamma^{2}, 1\right\}$. Hence, for some $K_{2}>0$ we have $|D(\omega)|>K_{2}$ for $\omega$ sufficiently large. Since $\left\|(\mathrm{j} \omega I-A)^{-1}\right\|$ and $|h(\mathrm{j} \omega)|$ are bounded for large $\omega$, by using (29)-(31) in (28) we obtain

$|K|^{2} \leqslant K_{3}\|z\|_{E}^{2}$

for some $K_{3}>0$. Hence, from (27), (35) and (36) it follows that

$$
|\omega C|^{2} \leqslant K_{4}\|y\|_{E}^{2}
$$

for some $K_{4}>0$. Since $P$ is a symmetric, positive-definite matrix, by using (32)-(37) in (14) we obtain the following for large $\omega$ :

$$
\|z\|_{E}^{2} \leqslant K_{5}\|y\|_{E}^{2}
$$

for some constant $K_{5}>0$. Hence it follows that

$$
\sup \left\|(\mathrm{j} \omega I-L)^{-1}\right\|_{E}<\infty
$$

$|\omega| \geqslant \Omega$

for $\Omega>0$ sufficiently large. Hence, (15) follows from (21) and (39). Therefore, it follows from Theorem 2 that the semigroup $T(t)$ generated by $L$ is exponentially stable.
Remark 1. Let us consider the system given by (1)-(2)

$$
\begin{aligned}
& u_{t t}(x, t)=u_{x x}(x, t), \quad u(0, t)=0, \quad u_{x}(1, t)=v(t), \\
& y(t)=u_{t}(1, t),
\end{aligned}
$$

where $v(\cdot)$ and $y(\cdot)$ are the (boundary control) input and measured output, respectively. For the analysis of such systems frequency domain concepts may also be used, see e.g. Hämäläinen and Pohjolainen (2000), Rebarber (1993, 1995), and Weiss and Curtain (1997). In this approach, the representation given by (40) cannot be directly used, since it is not in the abstract linear system form. System (40) can be put into the boundary control system form defined in Salamon (1987). Let us define the following spaces and operators:

$$
\begin{aligned}
& H=\left\{(\phi \psi)^{\mathrm{T}} \mid \phi \in \mathbf{H}_{0}^{1}, \psi \in \mathbf{L}^{2}\right\}, \\
& Z=\left\{(\phi \psi)^{\mathrm{T}} \mid \phi \in \mathbf{H}_{0}^{2}, \psi \in \mathbf{H}_{0}^{1}\right\}, \\
& \Delta w=\left(\psi \phi^{\prime \prime}\right)^{\mathrm{T}}, \quad \Gamma w=\phi^{\prime}(1), \quad K w=\psi(1),
\end{aligned}
$$

where $w=(\phi \psi)^{\mathrm{T}} \in Z$, and $D(\Delta)=Z$. For $w=\left(u u_{t}\right)^{\mathrm{T}}$, system (40) may be expressed as

$\dot{w}=\Delta w, \quad \Gamma w=v, \quad y=K w$,

for details, see Salamon (1987). It can be shown that the system given by (41)-(43) is well-posed (in the sense of Salamon), see Salamon (1987). If we take the Laplace transform of (40), after some straightforward algebra we obtain $\hat{y}(s)=g(s) \hat{v}(s)$ where $g(s)=(\sinh s) /(\cosh s)$. Under certain conditions, boundary control systems of form (43) may be expressed as an abstract linear system in the following form:

$\dot{w}=A w+B v, \quad y=C_{A} w+D v$

see Salamon (1987) for the relation between various operators and their domains in (43), (44). For a meaningful relation between (43) and (44), $D=\lim _{s \rightarrow \infty} g(s)$ should hold, and in the present case we have $D=1$. By using the well-posedness result stated above, the fact that $|D|<\infty$, and by using the results of Weiss (1994) and Weiss and Curtain (1997), it can be shown that representation (44) is well-posed and regular (in the sense given in Weiss \& Curtain, 1997). It is well-known that for system (43), with the static feedback law $v=-\alpha y$, $\alpha>0$, the resulting closed-loop system is exponentially stable. Either by using this result, or by direct calculation, it can be shown that representation (44) is stabilizable and detectable in the sense of Weiss and Curtain (1997), see Rebarber (1993, 1995) for similar calculations. By using these results, exponential stability result presented in this note may be obtained by using the frequency domain techniques, see e.g. Weiss and Curtain (1997). More precisely, consider the feedback law given by $\hat{v}(s)=-h(s) \hat{y}(s)$, where $h(s)$ is given by (6). Let us define $g(s)=C_{\Lambda}(s I-A)^{-1} B+D$, (which is $g(s)=(\sinh s) /(\cosh s)$ in our case $)$, and the feedback 
system transfer matrix $H(s)=\left[h_{i j}(s)\right]$ with $h_{11}=h_{22}=$ $(1+h g)^{-1}, h_{12}=h(1+h g)^{-1}, h_{21}=g(1+h g)^{-1}$, see Weiss and Curtain (1997). Then the exponential stability result presented in this note may be obtained by showing that $H(s) \in H^{\infty}$, i.e. its poles are in the open left-half of the complex plane, and $\sup _{\omega}\|H(\mathrm{j} \omega)\|<\infty$, see e.g. Proposition 4.6 of Weiss and Curtain (1997).

Remark 2. Most of the research in the area of boundary control of infinite-dimensional systems is concentrated on the problem of stabilization of conservative flexible structures (e.g. strings and beams without damping). Such systems have infinitely many poles on the imaginary axis and can be uniformly stabilized by using simple static controllers (e.g. the system given by (1)-(3)), see Chen (1979) and Luo, Guo, and Morgül (1999). It was known that these systems become unstable when arbitrary small time delays were introduced in the feedback law, see e.g. Logemann, Rebarber, and Weiss (1996). Although in the case of conservative systems (i.e. without damping), the use of dynamic controllers presented in this note will not change the non-robustness result stated above, when damped models are used certain improvements may be obtained by the use of dynamic controllers, see Morgül (1995).

\section{Conclusion}

In this note, we considered the stabilization of the wave equation in a bounded domain by means of a dynamic boundary control law. The transfer function of the controller may contain simple poles on the imaginary axis. This type of controllers were proposed for the stabilization of the wave equation, however only asymptotic stability results were given. In this note, we proved that with the proposed controller the closed-loop system is actually exponentially stable under some conditions.

\section{References}

Chen, G. (1979). Energy decay estimates and exact boundary value controllability for the wave equation in a bounded domain. Journal de Mathematiques Pures et Appliquees, 58, 249-273.

Curtain, R. F., \& Zwart, H. J. (1995). An introduction to infinite-dimensional linear systems theory. New York: Springer.

Hämäläinen, T., \& Pohjolainen, S. (2000). A finite-dimensional robust controller for systems in the CD-algebra. IEEE Transactions on Automatic Control, 45(3), 421-432.

Logemann, H., Rebarber, R., \& Weiss, G. (1996). Conditions for robustness and nonrobustness of the stability of feedback systems with respect to small delays in the feedback loop. SIAM Journal of Control and Optimization, 34, 572-600.

Luo, Z. H., Guo, B. Z., \& Morgül, Ö. (1999). Stability and stabilization of infinite dimensional systems with applications. London: Springer.

Morgül, Ö. (1994). A dynamic control law for the wave equation. Automatica, 30(11), 1785-1792.

Morgül, Ö. (1995). On the stabilization and stability robustness against small time delays of some damped wave equations. IEEE Transactions on Automatic Control, 40(9), 1626-1630.

Morgül, Ö. (1998). Stabilization and disturbance rejection for the wave equation. IEEE Transactions on Automatic Control, 43(1), 89-95.

Rebarber, R. (1993). Conditions for the equivalence of internal and external stability for distributed parameter systems. IEEE Transactions on Automatic Control, 38, 994-998.

Rebarber, R. (1995). Exponential stability of coupled beams with dissipative joints: A frequency domain approach. SIAM Journal of Control and Optimization, 33, 1-28.

Salamon, D. (1987). Infinite dimensional systems with unbounded control and observation: A functional analytic approach. Transactions of the American Mathematical Society, 300, 383-431.

Weiss, G. (1994). Transfer functions of regular linear systems, part 1: Characterizations of regularity. Transactions of the AMS, 342, 827-854.

Weiss, G., \& Curtain, R. F. (1997). Dynamic stabilization of regular linear systems. IEEE Transactions on Automatic Control, 42(1), $4-22$. 